\title{
IMPLEMENTACIÓN Y TRANSADAPTACIÓN DE ACTIVIDADES REVELADORAS DEL PENSAMIENTO (MEAS) EN LA ENSEÑANZA DE ESTADÍSTICA EN EL NIVEL MEDIO SUPERIOR Y A NIVEL SUPERIOR. UN CASO DE ESTUDIO
}

\author{
Guillermina Sánchez L. ${ }^{1}$, José D. Zacarías F. ${ }^{1}$, Gladys D. Salgado S. ${ }^{1}$ y Martha García Cruz ${ }^{2}$ \\ ${ }^{1}$ Benemérita Universidad Autónoma de Puebla, México \\ ${ }^{2}$ Universidad Politécnica de Amozoc, México \\ guisalop@hotmail.com
}

El presente estudio descriptivo-cualitativo aplicado a 150 estudiantes pretende demostrar la utilidad de las MEAs (Model-Eliciting Activities) en la enseñanza de la estadística en diferentes niveles educativos; para ello la actividad fue transadaptada al implementarse en cada grupo, manteniendo como propósito que alrededor de una problemática similar se aplique un análisis estadístico que ayude a plantear propuestas de solución. La MEA aplicada en el nivel medio superior aprovecha un suceso real: la BUAP, en el año 2014 presentó un cambio de imagen institucional basado en la idea de proyectar una presencia sólida y de gran peso, y de ahí surge la pregunta detonadora ¿Por qué la BUAP cambió su norma tipográfica? En el caso de la transadaptación para el nivel de ingeniería la problemática fue ficticia: «El dueño de un café internet cuya principal actividad es la impresión de documentos está casi en quiebra ¿Qué puede proponérsele para mejorar su situación?» En ambos casos se obtuvo un análisis estadístico similar.

\section{ANTECEDENTES}

La estadística es de vital importancia para el desarrollo de la sociedad debido a su utilidad en la investigación, la técnica y la vida profesional, pues permite una toma de decisiones eficiente en diversos ámbitos, como el social, económico y político, motivo por el cual el conocimiento de la estadística es muy relevante, pues ayuda a formar individuos que comprendan todas y cada una de las presentaciones y manejos de datos. En esta formación debe irse más allá de la simple transmisión de conocimientos, siendo necesario buscar la construcción del conocimiento por parte de los estudiantes paralograr un aprendizaje significativo donde, como dice Ausubel (1983) «se unan los conocimientos previos con los actuales para lograr el aprendizaje combinado que permitirá al estudiante apropiarse y por tanto aplicar los conocimientos adquiridos», y se posibilite la formulación, validación e institucionalización, siendo en este proceso, como menciona Batanero (1997), «importante el papel del docente, como un gestor del conocimiento y del medio».Por lo general, en las clases de matemáticas se tiene como problema la mecanización de los procesos, haciendo a un lado el desarrollo de la competencia matemática que, según el Programa Internacional de Evaluación de Estudiantes (PISA), se define como la capacidad para analizar, razonar y comunicar los resultados de una problemática real mediante la matematización (OECD, 2009), lo cual, como lo menciona Batanero (2000), hace necesario mostrar a los estudiantes no el carácter determinista de las matemáticas, sino una imagen real, lo que implica la resolución de problemas de la vida diariaa fin de no sólo resolver problemas, también de aumentar las capacidades de comunicación, tratamiento de la información y resolución de problemas recalcando, como menciona Begg (1997) citado por Batanero (2000), que la enseñanza de la estadística debe hacerse mediante una metodología heurística y activa para eliminar problemas encontrados en las sesiones de clase de esta materia, a saber:

- Los continuos cambios de contenido y de puntos de vista de las demandas de formación.

- El requerimiento social comprender las técnicas básicas de análisis de datos y de su interpretación.

- La rápida evolución de la estadística.

- El reducido número de investigaciones de didáctica de la estadística.

- La carencia de formación específica de profesores de estadística.

- La naturaleza interdisciplinaria de la estadística, pues aunque en muchas materias puede hacerse uso de ella, hay conflictos cuando las definiciones o propiedades presentadas no coinciden con las mostradas por el profesor de matemáticas. 
Resolver estos problemas permite alcanzar los fines de la enseñanza de la estadística, que según Batanero (2000) son:

- Que el estudiante comprenda y aprecie el papel de la estadística en todos los ámbitos de la sociedad, tomando en cuenta sus diferentes campos de aplicación y su influencia en el desarrollo.

- Que el estudiante conozca y aplique el razonamiento estadístico, sus alcances y limitantes.Inculcar en el estudiante la necesidad de la investigación estadística, debido al avance y cambio constante de esta ciencia, enfatizándoles que los fenómenos aleatorios tienen una fuerte presencia en nuestro entorno, como en los juegos de azar, las características genéticas, la previsión atmosférica, los resultados electorales, los análisis bioquímicos de las enfermedades y de las adicciones, por mencionar algunos.

Teniendo en cuenta lo anterior, en las clases de estadística es importante trabajar por proyectos, los cuales, como dice Valdés (2013), permiten a los estudiantes desarrollar capacidades como: seleccionar, recabar, codificar, analizar e interpretar datos; realizar investigación teórica y concluir sobre la aplicación de los datos en la vida real, a fin de modelar la solución a los problemas mostrando, como sugiere Holmes (1997) citado por Batanero (2000), que «la estadística no se reduce a contenidos matemáticos». Así, estos proyectos que «el estudiante los concibe como verdaderas investigaciones, donde trata de integrar la estadística dentro del proceso más general de la investigación», alcanzan el objetivo de dar una propuesta de solución. Cuando el estudiante llega a este punto se ha dado cognitivamente una apropiación de conocimientos que permite un aprendizaje más significativo.

Para este fin es deseable emplear actividades que generen esta comprensión. Las MEAs -por sus siglas en inglés (Model Eliciting-Activities), Actividades Reveladoras del Pensamiento-, como dice Chamberlain (2005), promueven la construcción del conocimiento a medida que se va estableciendo por parte de los estudiantes la resolución de las problemáticas planteadas, las cuales surgen a través de una pregunta detonadora que plantea una necesidad, misma que, al buscarse su solución permite la unión de los conocimientos previos con los actuales, provocando, como lo indican Lesh et al. (2000) «revelar, probar, refinar y extender el razonamiento». Al término del proceso los estudiantes deben entregar un informe dirigido a la persona que presentó la problemática, lo cual permite que cada valor estadístico halladotenga un sentido, ya que tiene que realizarse una interpretación del mismo y buscar su relación correspondiente para integrar la propuesta de solución, con lo que se produce un razonamiento mayor ya que no sólo se está desarrollando un procedimiento estadístico para obtener un resultado, sino que está haciéndolo para resolver una problemática real en particular, y tiene que explicarlo. Es importante recalcar que una MEA no tiene un sólo resultado numérico, ya que al requerir una propuesta de solución ésta puede darse a partir de diferentes enfoques. Es ahí donde se realiza la metacognición en los estudiantes.

En el diseño de las MEAS deben cumplirse, como lo proponen Lesh et al. (2000), 6 principios básicos: «Realidad, Construcción del modelo, Documentación, Autoevaluación, Reutilización y Prototipo eficaz». De ahí que en este trabajo la reutilización sea el motivo conductor en la comprobación de que, al transadaptar una MEA es posible aplicarla en diferentes niveles educativos.En este proceso es muy importante el uso de instrumentos de evaluación adecuados Si bien una MEA no tiene un resultado numérico único, es importante que el docente delimite el trabajo del estudiante para que sepa que se espera de él, qué aprendizajes debe aplicar y cómo debe ir desarrollándolos para escribir el informe final, cuyo contenido también debe ser perfectamente bien delimitado en la rúbrica.

\section{METODOLOGÍA}

En la aplicación de una actividad reveladora del pensamiento deben seguirse etapas bien definidas debido a la necesidad de desarrollar en los estudiantes habilidades y destrezas al momento de aplicar los conocimientos. En este proceso que no es fácil, el docente debe ser un guía que encamine a los estudiantes con este propósito, ya que una actividad reveladora del pensamiento no presenta una instrucción operacional única; al contrario, la problemática planteada puede ser 
resuelta por varios caminos, y es ahí donde el docente tiene que dirigir al estudiante para que llegue a resolverla aplicando los conocimientos de la materia en cuestión. Como resultado de la MEA los estudiantes elaboran un informe dirigido a la institución o a la persona que plantea la problemática. En el caso del presente trabajo, las fases en las cuales se realizó son las siguientes:

\section{Etapa 1. Selección de la muestra:}

Se seleccionaron 5 grupos (150 alumnos), distribuidos de la siguiente manera:2 grupos de la materia de «Estadística», en el 3. ${ }^{\text {er }}$ año del área propedéutica de Ciencias Sociales de la Preparatoria Regional «Simón Bolívar» de la Benemérita Universidad Autónoma de Puebla.

2 grupos de la materia «Control Estadístico de Procesos», dl 9. ${ }^{\circ}$ cuatrimestre de la Ingeniería en Automatización de la Universidad Tecnológica de Puebla .

1 grupo de la materia «Estadística y probabilidad», correspondiente al primer cuatrimestre de la de la Ingeniería en Software de la Universidad Politécnica de Amozoc.

\section{Etapa 2 Actividad de Enganche:}

Esta actividad tuvo como finalidad obtener un diagnóstico de cada grupo e interesarlos en el estudio de la estadística. La actividad se aplicó contextualizada según las características del grupo en cuestión, y fue propuesta con dos variaciones:

Prueba tipo A: Plantea la dificultad de elegir entre 4 candidatos posibles para obtener el premio al mejor promedio. En ella los datos numéricos presentados a los estudiantes aparentemente son diferentes, pero al comenzar a obtenerse los parámetros estadísticos correspondientes se observa que coinciden todos los candidatos con el mismo promedio - la moda - y también la mediana, con lo que se promueve la necesidad de que los estudiantes realicen una investigación para dar solución a la problemática planteada.

Prueba tipo B: Plantea que hay que escoger entre 4 alumnos reprobados a cuál aprobar. Al igual que en la prueba tipo A, media, mediana y moda no ayudan a llegar al resultado; al usar las medidas de dispersión el estudiante llega a profundizar en la conceptualización para la interpretación del resultado.

Como segunda parte se pidió a los estudiantes contestar unas preguntas de reflexión encaminadas a concientizarlos sobre la necesidad de desarrollar la habilidad matemática.

Al término de la actividad se observó que, del total de evaluados, $45 \%$ logró llegar a establecer la toma de decisión fundamentada para la solución del tipo de la Prueba A y 15\% lo hizo para la prueba B. Estos resultados nos permitieron comprobar que los estudiantes, en su mayoría, conciben a la estadística como la parte de las Matemáticas que se encarga de«sacar la media, mediana y moda de una serie de datos», lo cual provoca un problema enorme al tratar de analizar, razonar y modelar problemas para tomar decisiones y proponer soluciones. También se demuestra la necesidad de plantear, en las materias de estadística, actividades que ayuden a los estudiantes a adquirir y entender los conocimientos para poder aplicarlos correctamente.

\section{Etapa 3. Implementación de la MEA:}

En cada grupo se formaron equipos de 3 ó 4 personas y les fue entregada tanto la Rúbrica de Evaluación como la Actividad Reveladora del Pensamiento, la cual fue transadaptada para tener una mejor contextualización y aceptación por parte de los estudiantes dependiendo de la institución y grado donde se aplicó, lo cual es uno de los principios básicos para el uso de estas actividades. El orden en el que fueron implementadas fue el siguiente:

Para los 2 grupos de nivel medio superior de la Preparatoria Regional «Simón Bolívar» la MEA implementada es: ¿Por qué la BUAP cambió de tipografía?Para los 2 grupos de la Ingeniería en Automatización de la Universidad Tecnológica de Puebla y para el grupo de la Ingeniería en Software de la Universidad Politécnica de Amozoc la MEA aplicada es: ¿Es posible que el café internet «Cyber“, cuya principal actividad es la impresión de documentos, salga del problema económico en el que se encuentra?

En el planteamiento de la actividad se utilizó una sesión de una hora para informar y organizar a los estudiantes y entregar los instrumentos de evaluación. . Se acordó que el tiempo de entrega del informe resultante es de 2 semanas, teniendo por semana la posibilidad de ocupar una sesión de clase como tiempo para consultar sus dudas. 
Etapa 4. Grupos Focales:

Con la finalidad de unificar resultados se formó un equipo de cada grupo, mediante un muestreo aleatorio, para profundizar y reflexionar acerca de los resultados planteados. Es importante resaltar que esos equipos son los encargados de elaborar el informe final que unificará la aportación de cada grupo.

\section{ANÁLISIS DE RESULTADOS}

Las MEAs aplicadas son el resultado de un desarrollo de actividades a partir de las preguntas detonadoras: ¿Por qué la BUAP cambió de tipografia? y ¿Es posible que el café internet "Cyber", cuya principal actividad es la impresión de documentos salga del problema económico en el que se encuentra?

Aunque en un contexto diferente, ambas MEAS plantean el gasto de tinta que provoca imprimir con diferentes tipos de letra y si eso tiene un valor considerable que eleve el gasto mensual de una institución o de un negocio.

Se solicita que la modelación sea expresada mediante un informe de investigación dirigido al director de la institución o una carta dirigida al dueño del establecimiento, según sea el caso de la transadaptación.

Rúbricas: Parte importante de este trabajo son los instrumentos de evaluación que permiten determinar el nivel de capacidades y destrezas alcanzados por los estudiantes. En el caso de esta MEA se evaluaron:

- El contenido del reporte.

- La identificación del problema y el planteamiento de la solución.

- El planteamiento estadístico.

- La aplicación de la estadística.

- La fundamentación de propuestas.

- Los recursos utilizados en la investigación.

- La responsabilidad en los tiempos de entrega.

- El trabajo colaborativo.La investigación realizada

Todo lo anterior permite establecer un juicio de valor con respecto a si los estudiantes alcanzaron las competencias esperadas y se apropiaron del conocimiento impartido.

Líneas Estratégicas: Al resolver la MEA se tuvieron dos enfoques de inicio; en el primero se considera la comparación de las características tipométricas de las diferentes familias de letra seguido por el estudio del tipo de impresora y sus características. En el segundo enfoque va dirigido hacia el ahorro de tinta, lo que lleva a analizar la impresión y las fuentes tipográficas. Cabe resaltar que los parámetros antes mencionados fueron analizados estadísticamente por los estudiantes, realizando las comparaciones correspondientes.

Modelos matemáticos

Para resolver la problemática se encontró que más de la mitad utilizó un modelo gráfico y estadística descriptiva, $10 \%$ utilizó la estadística descriptiva de las encuestas aplicadas, $10 \%$ únicamente estadística descriptiva.

Modelos mentales: La Tabla 1 muestra los modelos mentales que los estudiantes propusieron en la resolución de la problemática planteada. Como puede apreciarse, hubo varias vertientes para encontrar la propuesta de solución.

Tabla 1. Modelos mentales

\begin{tabular}{|l|l|}
\hline \multicolumn{1}{|c|}{$\begin{array}{c}\text { Modelo mental de } \\
\text { elección }\end{array}$} & \multicolumn{1}{|c|}{ Descripción } \\
\hline $\begin{array}{l}\text { Diferencias en las } \\
\text { familias tipográficas }\end{array}$ & $\begin{array}{l}\text { Realizan y comparan estadísticamente la tipometría de diferentes } \\
\text { familias tipográficas. }\end{array}$ \\
\hline Tipo de impresora & $\begin{array}{l}\text { Analizan estadísticamente el desempeño de las impresoras usadas más } \\
\text { comúnmente, dependiendo las marcas. }\end{array}$ \\
\hline
\end{tabular}




\begin{tabular}{|l|l|}
\hline $\begin{array}{l}\text { Uso de software } \\
\text { especializado }\end{array}$ & $\begin{array}{l}\text { Analizan estadísticamente el software existente para determinar el } \\
\text { ahorro de tinta y optimizar el uso de las familias tipográficas. }\end{array}$ \\
\hline Tipo de tinta & Analizan estadísticamente la diferencia entre tinta líquida y en polvo. \\
\hline
\end{tabular}

\section{INTERPRETACIÓN Y CONCLUSIONES}

El Programa Educativo (PE): Bachillerato Universitario para la asignatura: Probabilidad, Estadística y Temas Selectos de Matemáticas con Código PR06 2301, Nivel: A03 de la Benemérita Universidad Autónoma de Puebla, señala que «el estudiante que sale de la preparatoria debe: Saber comprender: fenómenos, datos, y conceptos, para resolver problemas, mostrando una actitud positiva hacia su aprendizaje y sus implicaciones sociales en el trabajo colaborativo». De igual manera, los programas de estudio emitidos por la Coordinación de las Universidades Tecnológicas y Politécnicas hacen énfasis en que, como resultado del estudio de la materia el estudiante desarrollará la capacidad de analizar y determinar causas de problemas de calidad, y mediante la aplicación de las herramientas estadísticas básicas del control de calidad llevará a cabo una toma de decisiones oportuna y asertiva para lograr la mejora continua tanto en las organizaciones como en sus procesos. A estas expectativas, la Actividad Reveladora del Pensamiento logró aportar los conocimientos y experiencias esperados en el perfil de egreso de los estudiantes de ambos niveles educativos. Esto puede observarse ya que, como resultado de la implementación, se observó en los equipos - como indican Johnson et al. (2010) - que los estudiantes se dieron cuenta de las limitantes que tienen en cuanto a su conocimiento matemático, lo que los motivó, con ayuda del docente, a investigar para obtener la solución — tal como como Leavitt et al. (2010) sugieren-; una vez obtenida ésta, la dificultad que tuvieron los integrantes de los equipos fue la de expresar el resultado obtenido redactándolo en una carta, proceso que viene a fortalecer la metacognición.

Además, al evaluar las rúbricas para los diferentes equipos se observó que entre los procesos mentales que los estudiantes llevaron a cabo están: La investigación de fundamentos teóricos, discriminación de variables, recolección de datos, análisis estadístico dependiendo del nivel —en Ingeniería llegaron a determinar tipos de proceso y normas de calidad—, redactaron el informe correspondiente por grupo: reunieron información y elaboraron un trabajo grupal.Por todo lo anterior se muestra que las actividades reveladoras del pensamiento generan:

- Transversalidad curricular.

- Aplicación de contenidosDesarrollo de actitudes y valores.

En comparación con lo que exponen Domínguez (2009) y Aliprantis et al. (2003), los estudiantes revelaron un pensamiento formal intermedio, ya que sus modelos matemáticos fueron predominantemente estadísticos gráficos.

En ambas MEAS, 95\% pudo dar una óptima propuesta de solución y 5\% se aproximó a ella. La autoevaluación se realizó permitiendo que ellos mejoraran su planteamiento gracias a la retroalimentación en sesión plenaria, lo cual muestra la conveniencia del uso de las Actividades Reveladoras del Pensamiento (MEAS) en estadística para favorecer el perfil de egreso del estudiante.

Trabajar actividades con modelos mentales permite al docente elevar el razonamiento del alumno y, por tanto, aprovecharlo para lograr la adquisición y desarrollo de competencias matemáticas.

\section{REFERENCIAS}

Aliprantis, C. D. y Carmona, G. (2003). Introduction to an Economic Problem: A models and modeling perspective. En Lesh, R. y Doerr, H. (Eds.).

Ausubel, Novak-Hanesian (1983). Psicología Educativa: Un punto de vista cognoscitivo. 2." ed., Trillas, México.

Beyond Constructivism: models and modeling perspectives on mathematics problem solving, learning, and teaching [Versión electrónica]. (pp. 255-64). Mahwah, NJ: Lawrence Erlbaum.

Batanero, C., Estepa, A., and Godino, J. D. (1997). «Evolution of student's understanding of statistical association in a computer-based teaching environment». In J. Garfield and G. Burrill (Eds.), Research on the Role of Technology in Teaching and Learning Statistics. 1996 
IASE Round Table Conference Papers, (pp. 191-205). Voorburg: International Statistical Institute.

Batanero, C. (2000) Controversies around the role of statistical test in experimental research [Versión electrónica]. (pp. 78-80). Mathematical Thinking and Learning.

Batanero, C., \& Díaz C. (2011) Estadística con proyectos, ReproDigital. Facultad de Ciencias de la Educación, Universidad de Granada. Recuperado de http://www.gr.es/batanero/pages/ARTICULOS/Libroproyectos.pdf.

Chamberlin A. Moon Sidney (2005).Model-Eliciting Activities as a tool to develop and identify creatively gifted mathematicians». Journal of advanced academics, pp. 37-47.

Domínguez, A. (2009). Actividades reveladoras del pensamiento: más que una forma de aprendizaje activo. Ponencia presentada en el $10^{\circ}$ Congreso Nacional de Investigación Educativa, Veracruz, México.

Johnson, P, \& Laird, N. (1983). Mental Models: Towards a Cognitive Science of Language, Inference and Consciousness [Versión electrónica]. Cambridge, Massachusetts: Harvard University Press.

Johnson-Laird, P. N. (2010). Mental models and human reasoning. PNAS, CVII (43). 1824318250. Recuperado el 23 de febrero de 2015 de http://www.pnas.org/content/107/43/18243.full.pdf + html.

Leavitt, D., \& Ahn, C. (2010). A middle grade teacher's guide to Model Eliciting Activities. En Lesh, R., Galbraith, L., Haines, C. y Hurford, A. (Eds.), Modeling student's mathematical modeling competences [Versión electrónica] (pp. 353-364). DOI: 10.1007/978-1-44190561-1-30.

Lesh, R., Galbraith, L., Haines, C., \& Hurford, A. (Eds.), Modeling student's mathematical modeling competences [Versión electrónica] (pp. 353-364) DOI: 10.1007/978-1-4419-05611330 .

Lesh, R., Hoover, M., Hole, B., Kelly, A., \& Post, T. (2000). Principles for developing thought revealing activities for students and teachers. En A. Kelly, R. Lesh (Eds.), Research Design in Mathematics and Science Education. 591-646. Recuperado el 2 de febrero de 2015 de http://www.cehd.umn.edu/rationalnumberproject/00_2.html.

Organización para la Cooperación y el Desarrollo Económico. (2009). PISA 2009. Assessment Framework. Key competencies in reading, mathematics and science. [Versión electrónica]. París, Francia: OECD

Valdez V., \& Guzmán T., (2013) Aprendizaje de la Probabilidad y la estadística basada en competencias mediante las TICS [Versión electrónica] (pp.75-78) Centro Universitario Querétaro, Qro. 